DOI: $10.17516 / 1999-494 X-0229$

УДК 621.396 .7

\title{
Software and Hardware Simulation \\ of Radar System Signals \\ of the Vertical Sounding
}

\author{
Evgeniy N. Garina, Vasiliy N. Ratushnyak ${ }^{a}$, \\ Andrey B. Gladyshev a and Dmitriy I. Smirnov*b \\ ${ }^{a}$ Siberian Federal University \\ Krasnoyarsk, Russian Federation \\ ${ }^{b}$ National Research University \\ of Electronic Technology \\ Moscow, Russian Federation
}

Received 18.02.2020, received in revised form 28.02.2020, accepted 11.03.2020

Abstract. The article deals with the main requirements for the structure of radar signals, the features of their formation, and the software and hardware radar signal complex of the vertical atmospheric sounding. The hardware radar implementation based on the PXI modules made by National Instruments and Keysight is given. The software LabVIEW model performing formation and generation of radar pulses with different types of phase and amplitude phase modulation and their spectral analysis are presented.

Keywords: sensing system of vertical sounding, Doppler radar, vertical sounding radar signal, wind profilometer, atmospheric inhomogeneities, small-size antenna array.

Citation: Garin E.N., Ratushnyak V.N., Gladyshev A.B., Smirnov D.I. Software and hardware simulation of radar system signals of the vertical sounding, J. Sib. Fed. Univ. Eng. \& Technol., 2020, 13(3), 370-378. DOI: 10.17516/1999-494X-0229

(c) Siberian Federal University. All rights reserved

This work is licensed under a Creative Commons Attribution-Non Commercial 4.0 International License (CC BY-NC 4.0).

* Corresponding author E-mail address: oborona-81@ya.ru 


\title{
Программное и аппаратное моделирование \\ радиолокационных сигналов РЛС \\ вертикального зондирования
}

\author{
Е.Н. Гарин ${ }^{\mathrm{a}}$, В.Н. Ратушняка \\ А.Б. Гладышев ${ }^{a}$, Д.И. Смирнов ${ }^{\sigma}$ \\ ${ }^{a}$ Сибирский федеральныий университет \\ Российская Федераџия, Красноярск \\ ${ }^{6}$ Национальный исследовательский университет \\ «Московский институт электронной техники» \\ Российская Федеращия, Москва
}

\begin{abstract}
Аннотация. В статье рассмотрены основные требования к структуре радиолокационных сигналов (РЛС), особенности их формирования и программно-аппаратный комплекс РЛС вертикального зондирования атмосферы. Приведены аппаратная реализация РЛС на базе модулей PXI производства National Instruments и Keysight и программная модель LabVIEW, осуществляющие формирование и генерацию радиолокационных импульсов с различными видами фазовой, амплитудно-фазовой модуляции, а также их спектральный анализ.

Ключевые слова: радиолокационная станция вертикального зондирования, доплеровский радар, РЛСВЗ, ветровой профилометр, атмосферные неоднородности, малоразмерная антенная решетка.
\end{abstract}

Цитирование: Гарин, Е.Н. Программное и аппаратное моделирование радиолокационных сигналов РЛС вертикального зондирования / Е.Н. Гарин, В.Н. Ратушняк, А.Б. Гладышев, Д.И. Смирнов // Журн. Сиб. федер. ун-та. Техника и технологии, 2020. 13(3). С. 370-378. DOI: 10.17516/1999-494X-0229

\section{Введение}

Анализ состояния атмосферы является важнейшей задачей в области метеорологии. Информация о скорости и направлении ветра на разных высотах, величине турбулентности и атмосферном приливе необходима для обеспечения безопасности полетов авиации, а также безаварийного движения наземных и морских транспортных средств. В настоящее время для получения информации о динамике атмосферных движений используют сеть метеорологических станций, которые определяют стандартные метеорологические показатели у поверхности Земли. Для объектов особой важности (аэропорты, морские порты, научные консерватории) и особых зон экологического мониторинга, где необходимы комплексные исследования параметров нейтральной атмосферы до высот 100 км, включая трехмерный вектор скорости турбулентностей, мезосферные «серебристые» облака, а также электронную концентрацию ионосферы, необходимо использовать радиолокационные станции вертикального зондирования (мезосфера-стратосфера-тропосфера-радар), основанные на Френелевском и Брэгговском рассеянии от турбулентных образований, и отражения электромагнитных волн в различных слоях атмосферы. Временное положение отраженного сигнала и сдвиг по частоте, обусловленный эффектом Доплера, дают точную информацию о высоте и скорости перемещения атмосферных неоднородностей. Вместе с этим при разработке радиолокационной станции вертикального зондирования (РЛСВЗ) атмосферы возникает комплексная задача выбора частотно-кодовой структуры радиолокационных 
сигналов, построения в целом структуры и антенной системы РЛСВЗ. Перспективным подходом в обработке сигналов с низким отношением сигнал/шум в приложении к радиолокационной станции вертикального зондирования является применение специальных зондирующих сигналов и сложных шумоподобных сигналов (коды Баркера, М-последовательности), а также «смычки» сигналов с различными законами модуляции для компенсации боковых лепестков автокорреляционной функции при их последующей обработке [1-3].

\section{Программная реализация радиолокационных сигналов РЛСВ3}

Разработка компактных радиолокационных станций вертикального зондирования с фазированной малоэлементной решеткой обусловлена их низкой стоимостью, энергопотреблением, высокой мобильностью и требует решения серьезных проблем, связанных, прежде всего, с уменьшением энергетического потенциала из-за общего снижения отношения сигнал/шум на входе приемника, за счет уменьшения апертуры антенны и уменьшения числа передающих модулей. Указанное обстоятельство не позволяет применять стандартные алгоритмы подавления отражений от местных предметов и выделения на их фоне полезных сигналов. В подобных условиях наиболее перспективными методами считают адаптивную компенсацию мешающих сигналов с использованием череспериодных автокомпенсаторов или, с учетом достаточно низкой динамики принимаемых зондирующих сигналов, составление «карты местных предметов» $[4,5]$.

Одним из наиболее перспективных подходов к вопросу улучшения разрешающей способности радиолокационной станции вертикального зондирования при уменьшении размеров антенной решетки является использование многочастотных сигналов. В этом случае сигнал с меньшей частотой служит для устранения побочных интерференционных максимумов диаграммы направленности и разрешения фазовой неоднозначности сигнала на более высокой частоте. Основные проблемы, возникающие при реализации подобного подхода, - обеспечение когерентности сигналов и идентичности высокочастотного тракта на различных частотах. Когерентность сигналов можно обеспечить формированием многочастотных сигналов при использовании стандарта частоты и времени в качестве опорного генератора. Идентичность высокочастотного тракта на разных частотах обеспечивается применением специально разработанных СВЧ-элементов с улучшенными характеристиками - фазочастотной, группового времени запаздывания, а также высокоточной калибровкой тракта. Таким образом, применение этих подходов позволит реализовать гибкое управление диаграммой направленности и работать как в режиме пространственно-разнесенных антенн, так и в режиме доплеровского качающегося луча.

Для решения задач вертикального зондирования атмосферы и непосредственно формирования сигналов радиопередающим устройством совместно с малоэлементной фазированной антенной решеткой необходимо рассмотреть следующие требования к функциональным возможностям РЛСВ3:

1. Обеспечение формирования сетки рабочих частот в диапазоне 40-60 МГц с шагом 20 кГц.

2. Обеспечение формирования на выходе задающего генератора различных радиоимпульсов с длительностью от 1 до 50 мкс, с периодом следования от 20 мкс до 60 мс:

$$
-372 \text { - }
$$


- прямоугольный одиночный импульс;

- одиночный импульс с гауссовской формой огибающей;

- одиночный импульс с модифицированной гауссовской формой огибающей;

- пакет импульсов в виде дополнительного кода;

- пакет импульсов в виде кода Баркера, Голда и др.

3. Усилитель мощности должен обеспечивать неискаженное усиление радиоимпульсов, поступающих с выхода возбудителя.

4. Выходная мощность в импульсе не менее 1 кВт, средняя мощность определяется параметрами радиоимпульсов на входе.

5. Коэффициент стоячей волны нагрузки на выходе усилителя мощности менее 2,5.

6. Обеспечение возможности фазовых задержек в каждом канале малоэлементной решетки для электронного сканирования атмосферы.

На текущий момент для формирования радиолокационных сигналов с определенным видом модуляции в основном используют задающие векторные генераторы сигналов, для которых модулирующая последовательность формируется путем считывания из памяти или в реальном масштабе времени. Процесс формирования радиолокационного сигнала заключается в создании квадратурных составляющих, закон изменения во времени которых соответствует заданному виду модуляции.

Принцип генерации зондирующих сигналов заключается в следующем. ПЛИС вырабатывает цифровой код синфазной $I(t)$ и квадратурной $Q(t)$ составляющих зондирующего сигнала, этот код формируется по алгоритму, заданному в специальном программном модуле (subvi) LabVIEW или Matlab, что обеспечивает максимальную гибкость генерации заданных сигналов при программировании. Цифровой код сформированного сигнала переводится в аналоговый сигнал цифроаналоговым преобразователем (ЦАП). Синфазная и квадратурная составляющие аналогового сигнала с выхода ЦАП фильтруются фильтром низкой частоты (ФНЧ) и поступают на вход генератора сигналов произвольной формы. В векторном генераторе сигналов используется прямой перенос сигналов из основной полосы в радиочастотный диапазон. На его входы поступают синфазная и квадратурная составляющие модулирующей последовательности импульсного сигнала и непрерывный ВЧ-сигнал на несущей частоте с высокочастотного генератора, и далее с выхода сформированный импульсный ВЧ-сигнал идет на усилитель мощности.

Основой формирования цифрового кода модулирующей последовательности является ПЛИС. Использование CORDIC-процессора, который по аппаратным ресурсам идентичен конвейерному процессору и работает в режиме вращения, в то же время позволяет получить сразу квадратурные составляющие псевдослучайной последовательности навигационного сигнала $I(t)$ и $Q(t)[6]$.

CORDIC-процессор работает в соответствии с выражением

$$
\begin{aligned}
& x^{(i+1)}=x^{(i)}-d_{i} y^{(i)} 2^{-i}, \\
& y^{(i+1)}=y^{(i)}+d_{i} x^{(i)} 2^{-i}, \\
& z^{(i+1)}=z^{(i)}-d_{i} \tan ^{-1} 2^{-i},
\end{aligned}
$$


где $x=\cos (z) ; y=\sin (z) ; z-$ целевой угол; $d \in\{-1,1\}$.

Существует несколько основных архитектур ПЛИС, каждая из которых имеет преимущество либо по скорости работы, либо по простоте реализации. При разработке вычислительных архитектур, работающих на частотах 100-200 МГц, необходима конвейеризация вычислений. Несмотря на то что конвейерная архитектура имеет большие накладные расходы на выравнивание задержек вычислительных блоков, ее использование оправданно при обработке и преобразовании сигналов при решении радиолокационных задач. Для ускорения вычислений угла вращения вектора сигнала предварительно рассчитывают промежуточные значения $\tan ^{-1} 2^{-i}$ и сохраняют в регистрах. На каждой итерации проверяется знак промежуточного значения угла, далее выбирается приращение угла на следующей итерации конвейера. Конвейерная реализация позволяет вычислять значения $I(t)$ и $Q(t)$ за один такт и способна работать на частоте более 100 МГц.

Гибкая программная архитектура LabVIEW дает возможность запрограммировать в ПЛИС необходимый вид и модуляцию радиолокационных сигналов (BPSK, QPSK, QAM и другие модуляции) и далее произвести автоматическую компиляцию кода $v i$ LabVIEW в VHDL для реализации логики ПЛИС (рис. 1, 2).

Вместе с этим данная программная архитектура позволяет изменять все параметры генерируемых радиолокационных сигналов: длительность импульса, частоту, период повторения, вид модуляции, базу сигнала (рис. 3,4$)$.

\section{Аппаратная реализация радиолокационных сигналов РЛСВЗ}

Для сокращения времени и стоимости разработки аппаратной реализации целесообразно использовать ряд существующих модульных элементов оборудования. Например, модули PXI производства National Instruments, Keysight, такие как векторные генераторы, анализато-

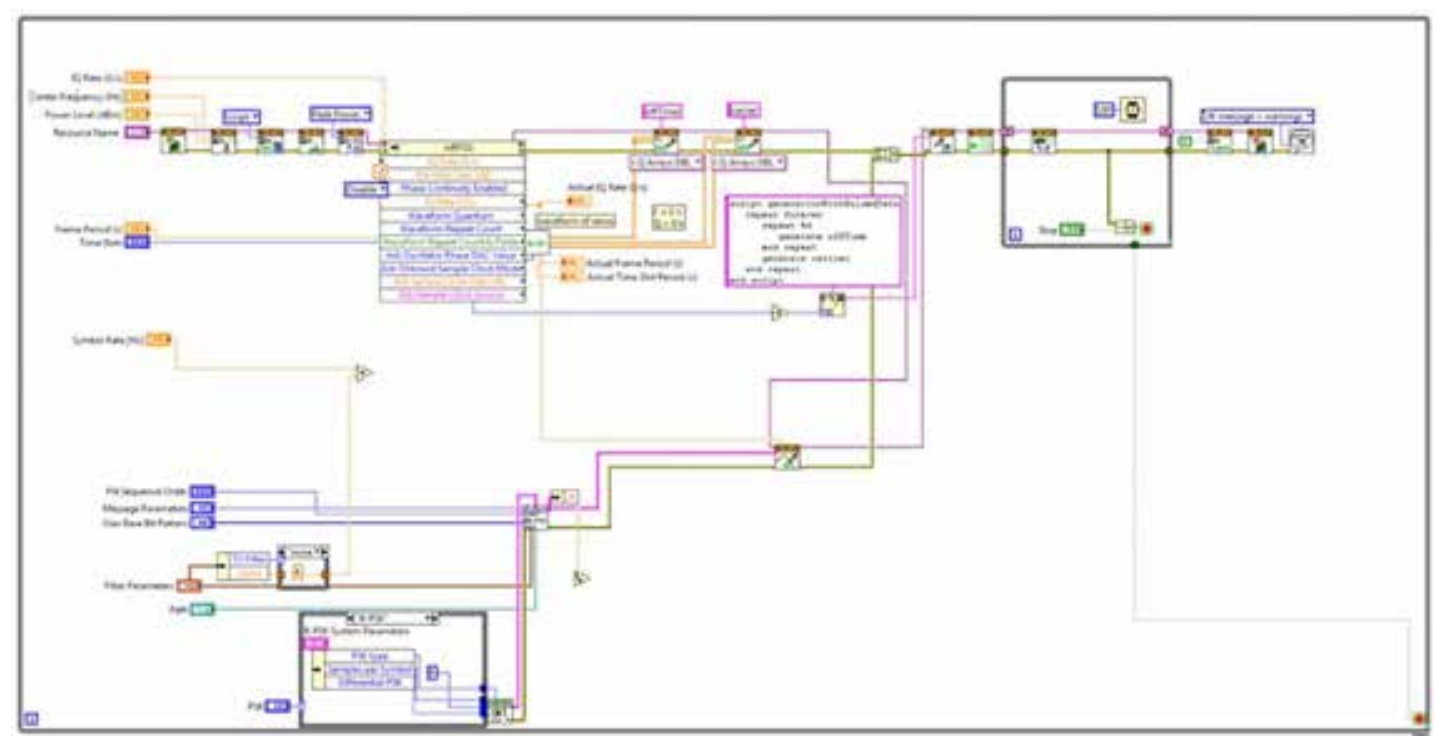

Рис. 1. LabVIEW VI блок-диаграмма формирования радиолокационного сигнала РЛСВ3

Fig. 1. LabVIEW VI block diagram formation of radar signal of the vertical sensing radar 


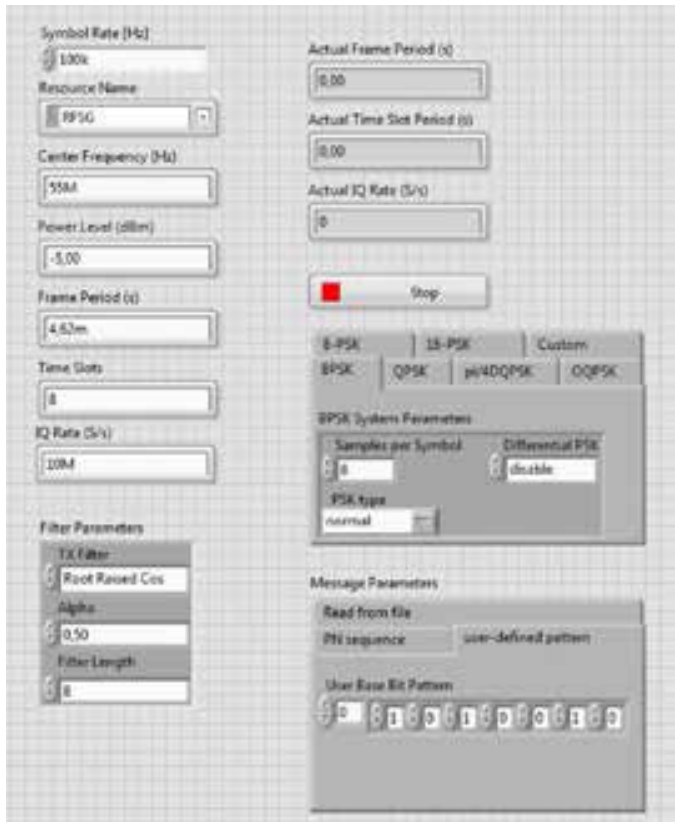

Рис. 2. LabVIEW VI фронт-панель управления сигналом РЛСВ3

Fig. 2. LabVIEW VI front panel signal management of the vertical sensing radar

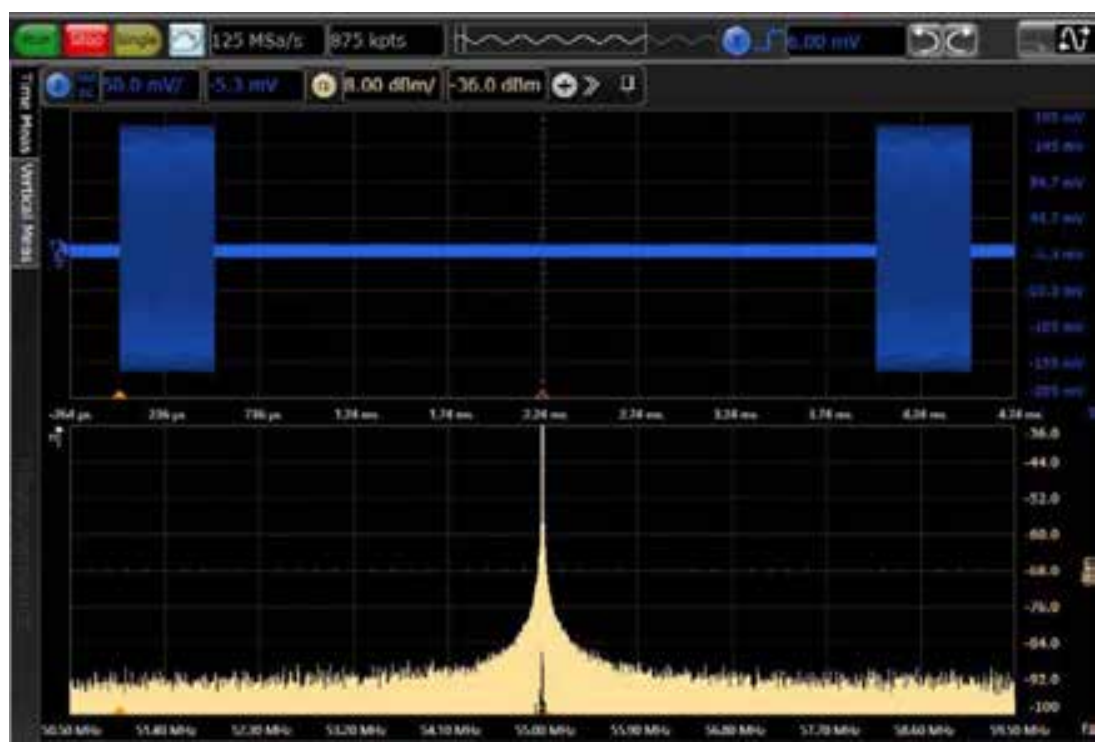

Рис. 3. Периодическая последовательность радиоимпульсов и их спектр РЛСВ3

Fig. 3. The periodic sequence of radio pulses and their spectrum of the vertical sensing radar

ры, программируемые ПЛИС, ЦАП и АЦП, которые полностью удовлетворяют требования по созданию радиолокационных сигналов, заданной структуры, их генерации в среду распространения и позволяют гибко управлять параметрами сигналов и в целом обеспечением зондирования различных слоев атмосферы. Генератор, выполненный на модулях PXI National 


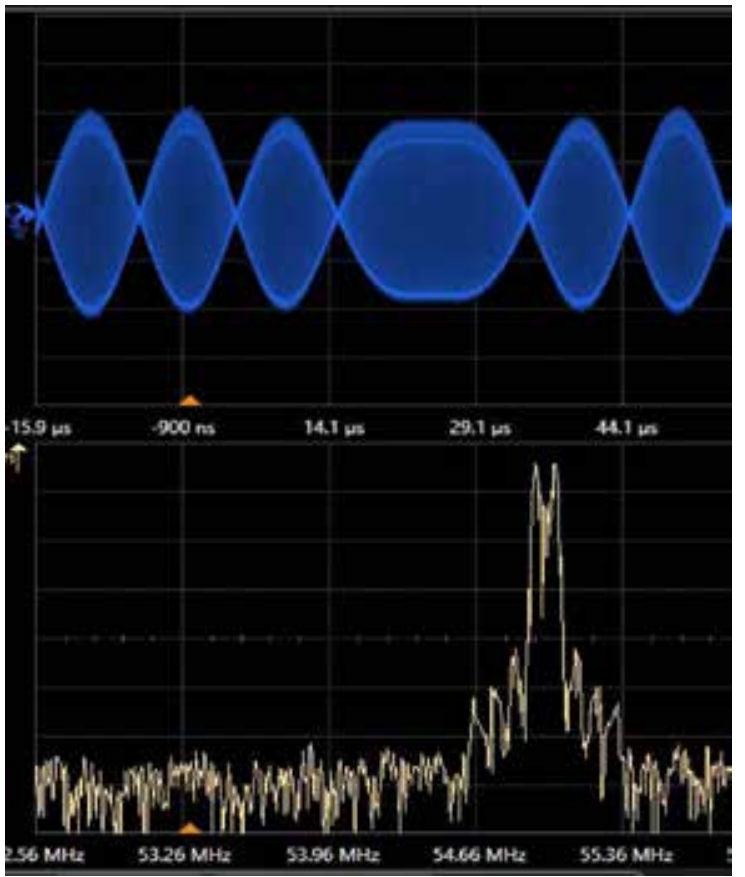

Рис. 4. Спектр и несущая 7-разрядного ФКМ сигнала РЛСВ3

Fig. 4. Spectrum and carrier of the 7-bit phase-code modulated signal of the vertical sensing radar

Instruments, содержит ПЛИС NI PXIe-7976R (Xilinx K710T), векторный генератор сигналов NI PXIe-5672, контроллер вычислительный NI PXIe-8880 (рис. 5).

Генератор, выполненный на модулях PXI Keysight, содержит генератор опорной частоты M9300A PXIе, синтезатор частот M9303А РХІе, цифровой векторный модулятор M9316А PXIе, предварительный усилитель М9312А, повышающий преобразователь частоты сигнала М9314А (рис. 6)

Принцип взаимодействия модулей векторного генератора сигналов Keysight приведен на блок-схеме (рис. 7).

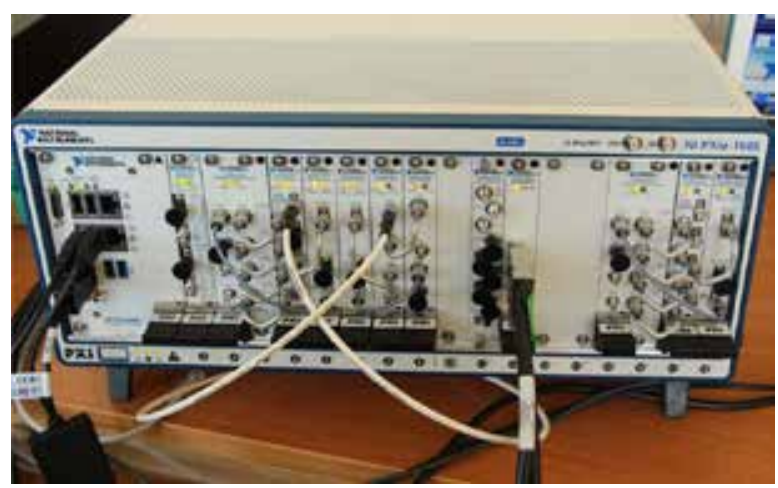

Рис. 5. Внешний вид задающего генератора на базе модулей PXI National Instruments

Fig. 5. Appearance of the generator based on the PXIe national Instruments chassis 


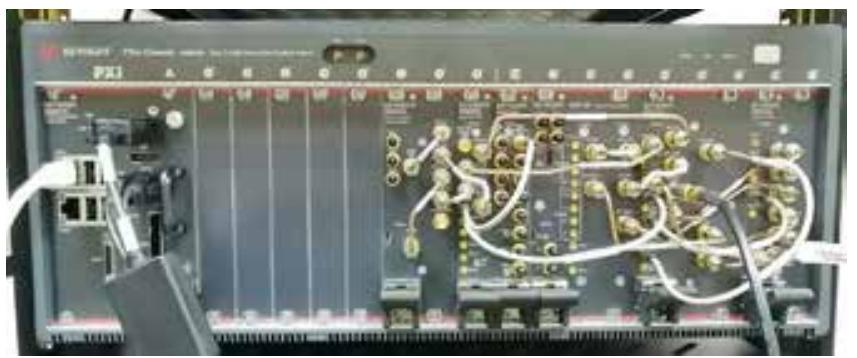

Рис. 6. Внешний вид задающего генератора на базе модулей PXI Keysight

Fig. 6. Appearance of the master generator based on the PXIe Keysight chassis

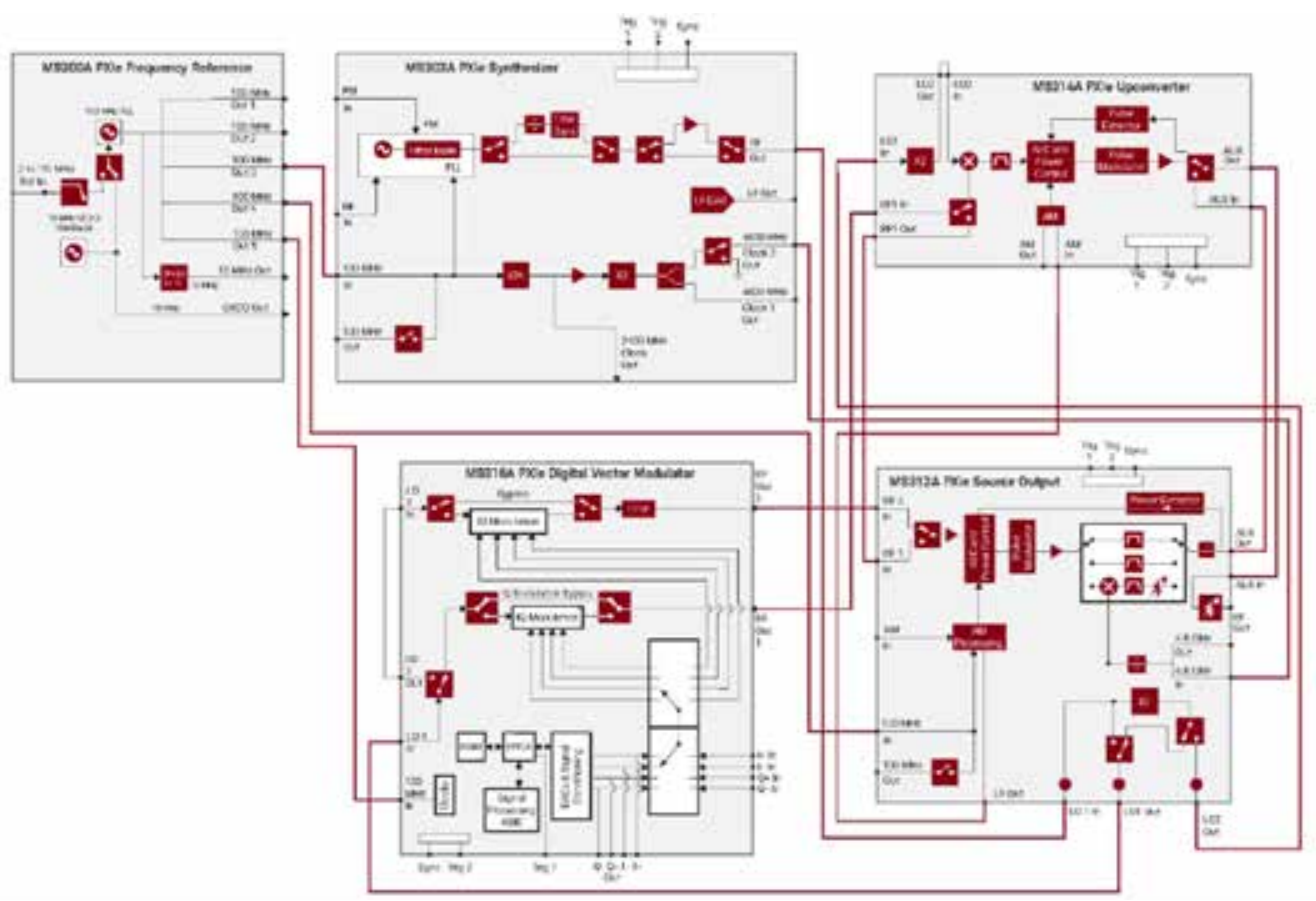

Рис. 7. Блок-схема М9383А (от 1 МГц до 44 ГГц)

Fig. 7. Block diagram M9383A (from $1 \mathrm{MHz}$ to $44 \mathrm{GHz}$ )

Данные программно-аппаратные решения на основе модульных элементов оборудования РХІ осуществляют формирование зондирующих сигналов с требуемыми параметрами и требуемым качеством частоты спектра.

\section{Заключение}

Таким образом, данные программно-аппаратные комплексы PXI National Instruments и Keysight позволяют генерировать радиоимпульсы с различной модуляцией с требуемым качеством. Гибкое управление модульными элементами программно-аппаратного комплекса дает возможность формировать заданную частотно-кодовую структуру, длительность, период повторения, мощность и частоту излучаемых радиолокационных сигналов. Такое управление

$$
-377-
$$


позволяет выполнить заданные характеристики РЛСВЗ (разрешающую способность по высоте, дальность зондирования, вероятность обнаружения, точность измерения неоднородностей атмосферы), а аппаратурная реализация с низкими массогабаритными показателями, энергопотреблением в сочетании с малоэлементной антенной решеткой обладает высокой мобильностью РЛСВЗ и помогает комплексно исследовать различные неоднородности атмосферы и нижней части термосферы, используя электронное сканирование.

\section{Благодарности / Acknowledgements}

Исследование выполнено при финансовой поддержке Российского фонда фундаментальных исследований, Правительства Красноярского края, Красноярского краевого фонда науки в рамках научного проекта 19-47-240002: «Методы радиолокационного зондирования атмосферы с использованием малоэлементных антенных решеток».

The reported study was funded by Russian Foundation for Basic Research, Government of Krasnoyarsk Territory, Krasnoyarsk Regional Fund of Science, to the research project: "Atmospheric radar sounding methods using low-element antenna arrays", ID no. 19-47-240002.

\section{Список литературы/References}

[1] Van Zandt T.E. A brief history of the development of wind-profiling or MST radars. Annales Geophysicae, 2000, 18(7), 740-749.

[2] Nishimura K., Nakamura T., Sato T., Sato K. Adaptive Beamforming Technique for Accurate Vertical Wind Measurements with Multichannel MST Radar. Journal of Atmospheric and Oceanic Technology, 2012, 29 (12), 1769-1775.

[3] Kashcheeva B., Proshkina E., Lagutin M. Remote methods and tools for studying processes in the Earth's atmosphere. Business Inform, 2002, 426.

[4] Vladimirov, V.M., Karmishin A.M., Markov V.V., Shepov V.N. Digital sounding-pulse generator for UHF MST radar. Conference Proceedings of 24th International Crimean Conference Microwave and Telecommunication Technology, 2014, 1119-1120.

[5] Владимиров В.М., Ратушняк В.Н., Вяхирев В.А., Тяпкин И.В. Особенности сканирования атмосферы и построения радиолокационных станций вертикального зондирования с малоэлементной антенной решеткой. Космические аппараты и технологии, 2019, 3(4), 237-242. [Vladimirov V.M., Ratushnyak V.N., Vyakhirev V.A., Tyapkin I.V. Features of scanning the atmosphere and building radar stations of vertical sounding with a low-element antenna array. Space vehicles and technologies, 2019, 3(4), 237-242. (in Russian)]

[6] Соколовский А.В., Высоцкий С.В., Ратушняк В.Н., Гладышев А.Б. Анализ эффективности VHDL-описаний архитектур сумматоров. Наукоемкие технологии, 2017, 18(12), 66-68. [Sokolovsky A.V., Vysotsky S.V., Ratushnyak V.N., Gladyshev A.B. Analysis of the effectiveness of VHDL descriptions of adder architectures. Knowledge-intensive technologies, 2017, 18(12), 66-68. (in Russian)] 